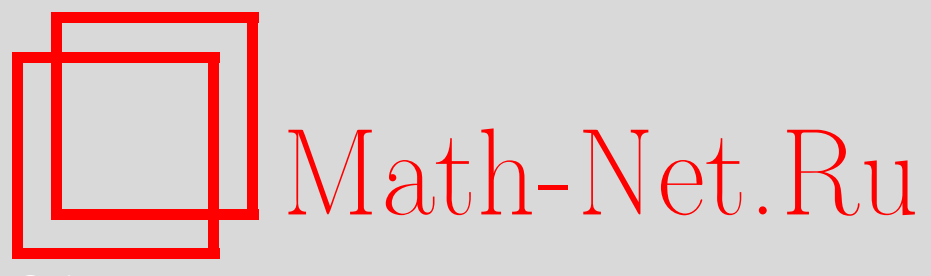

И. Н. Долгих, К. А. Мирзоев, Индексы дефекта и спектр самосопряженных расширений некоторых классов дифференциальных операторов, Матем. сб., 2006, том 197, номер 4, 53-74

DOI: https://doi.org/10.4213/sm1138

Использование Общероссийского математического портала Math-Net.Ru подразумевает, что вы прочитали и согласны с пользовательским соглашением http://www . mathnet.ru/rus/agreement

Параметры загрузки:

IP : 3.85 .183 .62

26 апреля 2023 г., 11:14:59

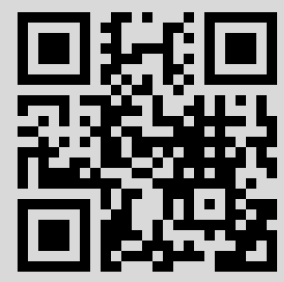




\author{
И. Н. Долгих, К. А. Мирзоев
}

\title{
Индексы дефекта и спектр самосопряженных расширений некоторых классов дифференциальных операторов
}

В настоящей работе исследуются задачи об асимптотическом поведении решений уравнения $l_{n} y=\lambda y$ произвольного порядка (четного или нечетного) с комплекснозначными коэффициентами в окрестности точки $+\infty$ и в окрестности нуля. При этом предполагается, что коэффициенты квазидифференциального выражения $l_{n}$ таковы, что если уравнение $l_{n} y=\lambda y$ свести к системе дифференциальных уравнений первого порядка, то полученную систему удастся преобразовать к системе дифференциальных уравнений с регулярной особой точкой при $x=\infty$ или $x=0$. Полученные результаты позволяют определить индексы дефекта соответствующих минимальных симметрических дифференциальных операторов и характер спектра самосопряженных расширений этих операторов.

Кроме того, с помощью уточненных асимптотических формул для решений уравнения $l_{n} y=\lambda y$ в работе найдены дефектные числа одного дифференциального оператора, порожденного дифференциальным выражением со старшим коэффициентом, обращающимся в нуль внутри рассматриваемого интервала.

Библиография: 14 названий.

\section{Введение}

Пусть комплекснозначные функции $f_{i j}, i, j=1,2 \ldots, n, n>1,-$ элементы матрицы-функции $F:=\left(f_{i j}\right)$ - определены на интервале $I:=(a, b),-\infty \leqslant a<$ $b \leqslant+\infty$, и удовлетворяют следующим условиям:

a) $f_{i j}=0$ почти всюду на $I$ при $2 \leqslant i+1<j \leqslant n$;

b) $f_{i j} \in \mathscr{L}_{1}(\alpha, \beta)$ для любых $\alpha, \beta \in I$ и $1 \leqslant i, j \leqslant n$, т.е. $f_{i j}$ локально абсолютно интегрируемы $\left(f_{i j} \in \mathscr{L}_{1, \mathrm{loc}}(I)\right)$;

c) $f_{i, i+1} \neq 0$ почти всюду на $I$ при $1 \leqslant i \leqslant n-1$;

d) $F=-J^{-1} F^{*} J$, где $F^{*}-$ сопряженная матрица к матрице $F$, т.е. $F^{*}=$ $\left(\bar{f}_{j i}\right)$, и $J:=\left((-1)^{i} \delta_{i, n+1-j}\right), 1 \leqslant i, j \leqslant n, \delta_{i j}-$ символ Кронекера.

Работа выполнена в рамках ведомственной научной программы Министерства образования и науки РФ "Развитие научного потенциала высшей школы" (проект “Спектральный анализ дифференциальных и разностных операторов", код 8351); кроме того, работа второго автора поддержана Российским фондом фундаментальных исследований (грант № 05-0100989) и Программой поддержки ведущих научных школ РФ (грант № НШ-1927.2003.1). 
Определим квазипроизводные заданной функции $y$ посредством матрицы $F$, полагая

$$
\begin{gathered}
y^{[0]}:=y, \\
y^{[i]}:=\left(f_{i, i+1}\right)^{-1}\left[\left(y^{[i-1]}\right)^{\prime}-\sum_{j=1}^{i} f_{i j} y^{[j-1]}\right], \quad i=1,2, \ldots, n-1,
\end{gathered}
$$

при условии, что $y^{[i-1]}$ уже определены и являются абсолютно непрерывными функциями на каждом компакте $[\alpha, \beta] \subset I$

$$
y^{[n]}:=\left(y^{[n-1]}\right)^{\prime}-\sum_{j=1}^{n} f_{n j} y^{[j-1]},
$$

и скалярное симметрическое квазидифференциальное выражение $l_{n}$, полагая

$$
l_{n} y:=i^{n} y^{[n]}
$$

Область определения выражения $l_{n}$ - это множество всех функций $y$, для которых существуют локально абсолютно непрерывные квазипроизводные $y^{[i]}$ до $(n-1)$-го порядка включительно. Для таких функций $l_{n} y$ существует почти всюду и локально интегрируемо на $I$.

Согласно общепринятой терминологии (см., например, [1; гл. IV, V], [2; гл. V] и $\left[3\right.$; гл. XIII]) выражение $l_{n}$ называется регулярным, если интервал $I$ конечен и элементы матрицы $F$ суммируемы на всем интервале $I$. В противном случае выражение $l_{n}$ называется сингулярным. При этом левый конец $a \in I$ интервала $I$ называется регулярным, если $a>-\infty$ и элементы матрицы $F$ суммируемы на каждом $[a, \beta] \subset I, \beta<b$. В противном случае конец $a$ называется сингулярнъм. Аналогично определяются понятия регулярности и сингулярности для правого конца интервала $I$.

Отметим, что условия а), с) и d) нужны для того, чтобы можно было определить скалярное симметрическое квазидифференциальное выражение $l_{n}$, порожденное матрицей $F$, а условие b) - для обеспечения справедливости теоремы существования и единственности решений соответствующих систем дифференциальных уравнений первого порядка.

Если элементы матрицы $F$ удовлетворяют условиям а) $-\mathrm{d})$, то множество финитных функций из области определения выражения $l_{n}$ образует всюду плотное линейное многообразие в гильбертовом пространстве $\mathscr{L}_{2}(I)$. Исходя из этого известным образом определяется минимальный замкнутый симметрический оператор $L_{0}$ в гильбертовом пространстве $\mathscr{L}_{2}(I)$, порожденный выражением $l_{n}$ (подробности см. [1; гл. II], [2; гл. V, § 17], [4]).

Обозначим через $n_{+}$и $n_{-}$дефектные числа оператора $L_{0}$ в верхней и нижней полуплоскости соответственно. В абстрактной теории симметрических операторов пару $\left(n_{+}, n_{-}\right)$называют индексом дефекта оператора $L_{0}$.

Известно, что если элементы матрицы $F$ удовлетворяют условиям а) - d) и один из концов интервала $I$ регулярный, то при любом невещественном $\lambda$ уравнение

$$
l_{n} y=\lambda y
$$


имеет решения из $\mathscr{L}_{2}(I)$ и $n_{+}\left(n_{-}\right)$равно максимальному числу линейно независимых решений уравнения (1) при $\operatorname{Im} \lambda>0(\operatorname{Im} \lambda<0)$, принадлежащих пространству $\mathscr{L}_{2}(I)$, и не зависит от $\lambda$.

Известно (см., например, [4]), что если матрица $F$ удовлетворяют условиям а)-d), то, в частности, справедлива

ТЕОрема 1. Числа $n_{+}$u $n_{-}$удовлетворяют следующим неравенствам:

1) если $n=2 m, m \geqslant 1, m o$

$$
\frac{1}{2} n=m \leqslant n_{+}, n_{-} \leqslant 2 m=n ;
$$

2) если $n=2 m+1, m \geqslant 1, m o$

$$
\begin{aligned}
\frac{1}{2}(n-1)=m & \leqslant n_{+} \leqslant 2 m+1=n, \\
\frac{1}{2}(n+1)=m+1 & \leqslant n_{-} \leqslant 2 m+1=n .
\end{aligned}
$$

Уравнение (1) равносильно системе линейных дифференциальных уравнений первого порядка

$$
\mathbf{y}^{\prime}=(F+\Lambda) \mathbf{y}
$$

где $\mathbf{y}:=\operatorname{colon}\left(y^{[0]}, y^{[1]}, \ldots, y^{[n-1]}\right)$, а элементы числовой матрицы $\Lambda:=\left(\lambda_{i j}\right)$ определяются равенствами $\lambda_{n 1}:=i^{-n} \lambda$ и $\lambda_{i j}:=0$ для остальных значений $i$ и $j$. Эквивалентность (1) и (2) понимается в том смысле, что если $y$ является решением (1), то $\mathbf{y}=\operatorname{colon}\left(y^{[0]}, y^{[1]}, \ldots, y^{[n-1]}\right), y=y^{[0]}$, является решением (2), и наоборот, если $\mathbf{y}=\operatorname{colon}\left(y_{0}, y_{1}, \ldots, y_{n-1}\right)-$ решение системы $(2)$, то $y=y_{0}-$ решение уравнения (1) и $y_{k}=y^{[k]}, 0 \leqslant k \leqslant n-1$ (более подробно см. [1], [2; гл. V], [3; гл. ХІІІ], [4]).

Следуя работе [5], рассмотрим один частный случай выражения $l_{n}$, а именно, предположим, что

I) функции $p_{0}(x), p_{1}(x), \ldots, p_{m}(x)$ измеримы, принимают вещественные значения при $x \in[1,+\infty)$ и при любом $1<b<+\infty$

$$
\int_{1}^{b}\left|p_{m}\right|^{-1}<+\infty, \quad \int_{1}^{b}\left|p_{k}\right|<+\infty, \quad k=0,1, \ldots, m-1
$$

II) $p_{0}(z), p_{1}(z), \ldots, p_{m}(z)$ - аналитические функции при $|z| \geqslant x_{0}>1$ и

$$
p_{k}(z):=z^{2 k+\nu}\left[a_{k}+\sum_{j=1}^{+\infty} a_{j}^{(k)} z^{-j}\right], \quad k=0,1, \ldots, m, \quad|z| \geqslant x_{0}>1
$$

где $a_{m} \neq 0$, а $\nu \geqslant 0$ - целое число.

Условие I) позволяет определить выражение $l_{n}$ порядка $n=2 m$ формулой

$$
\begin{aligned}
l_{2 m} y:= & p_{0}(x) y+\frac{d}{d x}\left\{p_{1}(x) y^{\prime}+\frac{d}{d x}\left[p_{2}(x) y^{\prime \prime}+\cdots\right.\right. \\
& \left.\left.+\frac{d}{d x}\left(p_{m-1}(x) y^{(m-1)}+\frac{d}{d x}\left(p_{m}(x) y^{(m)}\right)\right) \ldots\right]\right\}
\end{aligned}
$$


а из условия II) следует, что выражение $l_{2 m}$ при $x>x_{0}$ имеет вид

$$
l_{2 m}[y]=\sum_{k=0}^{m}\left(p_{k}(x) y^{(k)}\right)^{(k)} .
$$

Сформулируем теорему, принадлежащую С. А. Орлову (см. [5]).

ТЕОРема 2. Если функиии $p_{0}(x), p_{1}(x), \ldots, p_{m}(x)$ удовлетворяют условиям I) и II), то максимальное число линейно независимых решений уравнения (1), принадлежащих $\mathscr{L}_{2}(1,+\infty)$, равно

1) $п р и \nu>0$ числу корней полинома

$$
\mathfrak{F}_{2 m}(z, \nu):=\sum_{k=1}^{m} a_{k} \prod_{j=0}^{k-1}\left[\left(z+\frac{\nu}{2}\right)^{2}-\left(\frac{\nu+1}{2}+j\right)^{2}\right]+a_{0},
$$

лежащих в области $\operatorname{Re} z<0$, и не зависит от $\lambda$;

2) при $\nu=0$ числу корней полинома $\mathfrak{F}_{2 m}(z, 0)-\lambda$, лежащих в области $\operatorname{Re} z<0$, и при невещественном $\lambda$ равно $\mathrm{m}$.

При этом в случае $\nu>0$ спектр любого самосопряженного расширения оператора $L_{0}$ дискретный.

Предположим, что коэффициенты $p_{0}, p_{1}, \ldots, p_{m}$ выражения $l_{2 m}$ представляются в виде

$$
p_{k}(x)=x^{2 k+\nu}\left(a_{k}+r_{k}(x)\right), \quad k=0,1, \ldots, m-1, \quad p_{m}(x)=\frac{x^{2 m+\nu}}{1 / a_{m}+r_{m}(x)},
$$

где $\nu$ - неотрицательное (необязательно целое) число, $a_{0}, a_{1}, \ldots, a_{m}$ - вещественные числа, $a_{m} \neq 0$, а $r_{0}, r_{1}, \ldots, r_{m}$ - вещественные функции на $[1,+\infty)$. Пусть далее выполняются условия:

i) при некотором $x_{0}(\geqslant 1)$

$$
\int_{x_{0}}^{+\infty}\left|r_{k}(x)\right| \frac{d x}{x}<+\infty, \quad k=0,1, \ldots, m
$$

ii) все корни полинома $\mathfrak{F}_{2 m}(z, \nu)$ при $\nu>0$ и полинома $\mathfrak{F}_{2 m}(z, 0)-\lambda$ при $\nu=0$ различны.

В работе [6] при выполнении условий I), i) и іi) найдены асимптотические формулы для фундаментальной системы решений уравнения (1). В частности там доказано, что утверждения 1) и 2) теоремы 2 остаются справедливыми, если в ней условие II) заменить условиями і) и іi).

Отметим, что в [2] в общих чертах изложены содержания работ [5] и [6] (см. [2; гл. V, §17]). Работа [5] включена также в библиографию книги [3]. Однако в гл. XIII книги [3], посвященной обыкновенным дифференциальным операторам, нет ссылки на [5]. В работе [7], вошедшей позже в [8], заново открыта теорема 2 для частного случая $p_{k}(x)=a_{k} x^{2 k+\nu}, k=0,1, \ldots, m$, и подробно изучен полином $\mathfrak{F}_{2 m}(z, \nu)$. Таким образом, по-видимому, работы [5] и [6] так и остались незамеченными авторами книги [8]. В [8] рассматриваются 
и некоторые дифференциальные выражения нечетного порядка частного вида и исследуются соответствующие им полиномы.

Отметим также, что в [9; гл. 7] исследуются дифференциальные операторы, порожденные обыкновенными дифференциальными выражениями на конечном отрезке с двумя сингулярными концами.

Предположим теперь, что коэффициент при производной порядка $k$ дифференциального выражения $l_{n}$ имеет непрерывные производные до $k$-го порядка включительно на множестве $I$. Как известно, всякое симметрическое дифференциальное выражение порядка $n>1$ с такими коэффициентами можно представить в виде

$$
l_{n} y=\sum_{k=0}^{[n / 2]}\left(p_{k} y^{(k)}\right)^{(k)}+i \sum_{k=0}^{[(n-1) / 2]}\left\{\left(q_{k} y^{(k+1)}\right)^{(k)}+\left(q_{k} y^{(k)}\right)^{(k+1)}\right\},
$$

где $[a]$ - наибольшее целое число, не превосходящее числа $a$, а $p_{0}, p_{1}, \ldots, p_{[n / 2]}$ и $q_{0}, q_{1}, \ldots, q_{[(n-1) / 2]}$ - вещественнозначные функции на $I$ и

$$
p_{k} \in C^{(k)}(I), \quad k=0,1, \ldots,\left[\frac{n}{2}\right], \quad q_{k} \in C^{(k+1)}(I), \quad k=0,1, \ldots,\left[\frac{n-1}{2}\right] .
$$

При этом известен также вид матрицы $F$, посредством которой определяются квазипроизводные и квазидифференциальное выражение, совпадающее с выражением $l_{n}$ при выполнении условий (3) (см. [1; добавление A], [4], эти матрицы мы приводим также в 1$)$. Настоящая работа посвящена исследованию дифференциальных операторов, порожденных квазидифференциальными выражениями, определяемыми матрицами этого вида.

Первый параграф этой работы носит в основном вспомогательный характер и посвящен преобразованию системы (2) к виду, удобному для дальнейших исследований. Далее, в $\S 2$ и $\S 3$ исследуется задача об асимптотическом поведении решений уравнения (1) произвольного порядка (четного или нечетного) с комплекснозначными коэффициентами в окрестности точки $+\infty$ и правосторонней окрестности нуля и полученные результаты применяются к задаче об определении индекса дефекта соответствующих минимальных симметрических дифференциальных операторов и к задаче о характере спектра самосопряженных расширений этих операторов. При этом предполагается, что коэффициенты квазидифференциального выражения $l_{n}$ и, следовательно, элементы матрицы $F$ удовлетворяют условиям, аналогичным либо I) и II) $(\S 2$, теоремы 3,4$)$, либо I), i) и іi) (§3, теоремы 5-8) в окрестности соответствующей точки. Особо отметим, что эти условия обеспечивают, в частности, выполнение условий а)-d) для соответствующей матрицы $F$.

Теперь вкратце обсудим случай, когда коэффициенты выражения $l_{n}$ удовлетворяют условию (3), но старший коэффициент этого выражения (т.е. функция $p_{m}$ при $n=2 m$ или функция $q_{m}$ при $n=2 m+1$ ) обращается в нуль в некоторых точках внутри множества $I$. Как мы уже отмечали, и в этом случае можно построить матрицу $F$ и определить квазипроизводные заданной функции. Однако при этом для матрицы $F$ условие b) нарушается. Тем не менее множество бесконечно дифференцируемых финитных функций является всюду плотным линейным многообразием в пространстве $\mathscr{L}_{2}(I)$ и $l_{n} y \in \mathscr{L}_{2}(I)$, 
если $y$ - из этого множества. Далее, исходя из этого известным образом определяется минимальный замкнутый симметрический оператор $L_{0}$ в гильбертовом пространстве $\mathscr{L}_{2}(I)$, порожденный выражением $l_{n}$ (см. [3; гл. XIII, §2]).

В настоящей работе, следуя [3; гл. ХІІІ], будем называть дифференциальное выражение $l_{n}$ иррегулярным дифференциалъным выражением, если коэффициенты этого выражения удовлетворяют условию (3) и его старший коэффициент обращается в нуль в некоторых точках множества $I$.

Дифференциальные операторы, порожденные иррегулярными дифференциальными выражениями, возникают во многих областях современного анализа; таковыми являются, например, обобщенные операторы Лежандра (см. [9; гл. 7]) из теории квазиклассических ортогональных многочленов. Однако вопросы спектрального анализа этих операторов изучены удивительно мало. В частности, лишь сравнительно недавно стали появляться работы, посвященные определению дефектных чисел операторов, порожденных одночленными иррегулярными дифференциальными выражениями с вещественными коэффициентами (см. [10] и работы, на которые там имеются ссылки).

Итак, в работе [10] рассматривается минимальный симметрический дифференциальный оператор $L_{0}^{(p)}$, порожденный иррегулярным дифференциальным выражением

$$
\left(l_{2 m} y\right)(x):=\left(x^{p} a(x) y^{(m)}(x)\right)^{(m)}, \quad x \in(-1,1),
$$

где $p \in\{1,2, \ldots, 2 m-1\}, a(x)$ - бесконечно дифференцируемая действительная функция и $a(x) \neq 0$ для любого $x \in[-1,1]$. В ней доказано, что для верхнего дефектного числа $n_{+}\left(=n_{-}\right)$оператора $L_{0}^{(p)}$ справедлива формула $n_{+}=2 m+p$, если $p \in\{1,2, \ldots, m\}$. Кроме того, в [10] сформулирована гипотеза о справедливости равенства $n_{+}=4 m-p$ в случае $p \in\{m+1, m+2, \ldots, 2 m-1\}$. В $\{4$ настоящей работы, уточняя формулы для решений уравнения $\left(l_{2 m} y\right)(x)=\lambda y(x)$, $x \in(0,1]$, приведенные в $\S 2$ для более общего случая, мы находим дефектные числа оператора $L_{0}^{(p)}$, в частности доказываем справедливость сформулированной выше гипотезы.

Часть результатов (теоремы 4 и 6) настоящей работы опубликованы без доказательства в [11].

\section{§ 1. Преобразование основного уравнения}

Этот параграф посвящен преобразованию системы (2) к виду, удобному для дальнейших исследований. Здесь символом $I$ будем обозначать либо полуинтервал $(0,1]$, либо полуось $[1,+\infty)$.

Сначала рассмотрим случай $n=2 m$.

Известно, что при $m=1$ квазидифференциальное выражение $l_{2}$ порождается матрицей

$$
F_{2}:=\left(\begin{array}{cc}
-i q_{0} p_{1}^{-1} & -p_{1}^{-1} \\
p_{0}+q_{0}^{2} p_{1}^{-1} & -i q_{0} p_{1}^{-1}
\end{array}\right),
$$

где $p_{0}, q_{0}, p_{1}$ - вещественные функции на $I, p_{1}^{-1}, q_{0} p_{1}^{-1}, p_{0}+q_{0}^{2} p_{1}^{-1}$ из $\mathscr{L}_{1, \mathrm{loc}}(I)$, и имеет вид

$$
l_{2}=\left(p_{1} y^{\prime}+i q_{0} y\right)^{\prime}+p_{0} y+i q_{0} y^{\prime} .
$$


Пусть выполнено

Условие $\mathrm{A}(n=2 m)$. Функции $p_{0}, p_{1}, \ldots, p_{m}, q_{0}, q_{1}, \ldots, q_{m-1}, m=2,3, \ldots$, вещественные на I и все функции

$$
\begin{aligned}
\alpha_{m}:=(-1)^{m-1} p_{m}^{-1}, \\
\alpha_{m-1}:=(-1)^{m+1}\left(p_{m-1}+q_{m-1}^{2} p_{m}^{-1}\right), \quad \beta_{m-1}:=-q_{m-1} p_{m}^{-1}, \\
\alpha_{k}:=(-1)^{m+1} p_{k}, \quad \beta_{k}:=(-1)^{m+1} q_{k}, \quad k=0,1, \ldots, m-2,
\end{aligned}
$$

принадлежат $\mathscr{L}_{1, \mathrm{loc}}(I)$.

Определим матрицу $F_{2 m}:=F$, положив

$$
F_{2 m}=\left(\begin{array}{cccccccccccc}
0 & 1 & 0 & 0 & . & . & . & . & . & . & 0 & 0 \\
0 & 0 & 1 & 0 & . & . & . & . & . & . & 0 & 0 \\
0 & 0 & 0 & 1 & . & . & . & . & . & . & 0 & 0 \\
. & . & . & . & . & . & . & . & . & . & . & . \\
. & . & . & . & 0 & 1 & 0 & 0 & . & . & . & . \\
. & . & . & . & 0 & i \beta_{m-1} & \alpha_{m} & 0 & . & . & . & . \\
. & . & . & . & i \beta_{m-2} & \alpha_{m-1} & i \beta_{m-1} & 1 & . & . & . & . \\
. & . & . & . & \alpha_{m-2} & i \beta_{m-2} & 0 & 0 & . & . & . & . \\
0 & i \beta_{1} & \alpha_{2} & . & . & . & . & . & . & . & 1 & 0 \\
i \beta_{0} & \alpha_{1} & i \beta_{1} & . & . & . & . & . & . & . & 0 & 1 \\
\alpha_{0} & i \beta_{0} & 0 & . & . & . & . & . & . & . & 0 & 0
\end{array}\right)
$$

Таким образом, элементы матрицы $F_{2 m}$ имеют следующий вид:

$$
\begin{array}{ll}
f_{2 m-k, k+1}=\alpha_{k}, & k=0,1, \ldots, m ; \\
f_{2 m-k, k}=f_{2 m+1-k, k+1}=i \beta_{k-1}, & k=1,2, \ldots, m ; \\
f_{k, k+1}=1, & k=1,2, \ldots, m-1, m+1, \ldots, 2 m-1,
\end{array}
$$

и $f_{i j}=0$ при всех остальных значениях $i$ и $j$. Следовательно, матрица $F_{2 m}$ удовлетворяет условиям а)-d), если выполнено условие А.

Квазидифференциальное выражение $l_{2 m}, m \geqslant 1$, порожденное матрицей $F_{2 m}$, обладает тем свойством, что если $p_{k} \in C^{(k)}(I), k=0,1, \ldots, m, q_{k} \in C^{(k+1)}(I)$, $k=0,1, \ldots, m-1$ (см. (3)), и $p_{m}(x) \neq 0$ для всех $x \in I$, то оно совпадает с классическим дифференциальным выражением

$$
l_{2 m} y=\sum_{k=0}^{m}\left(p_{k} y^{(k)}\right)^{(k)}+i \sum_{k=0}^{m-1}\left[\left(q_{k} y^{(k+1)}\right)^{(k)}+\left(q_{k} y^{(k)}\right)^{(k+1)}\right]
$$

(см. [1; добавление А]).

Если же условие (3) выполняется, но $p_{m}(x)=0$ при некоторых $x \in I$, то $l_{2 m} y$ уже является иррегулярным дифференциальным выражением. Однако подчеркнем еще раз - и в этом случае выражение $l_{2 m} y$ порождается матрицей $F_{2 m}$ и определение квазипроизводных для этого выражения посредством матрицы $F_{2 m}$ остается в силе. 
Пусть выполнено

УСловие В $(n=2 m)$. Функции $p_{0}, p_{1}, \ldots, p_{m}, q_{0}, q_{1}, \ldots, q_{m-1}$ имеют вид $p_{k}(x):=x^{2 k+\nu}\left(a_{k}+r_{k}(x)\right), \quad q_{k}(x):=x^{2 k+\nu+1}\left(b_{k}+s_{k}(x)\right), \quad k=0,1, \ldots, m-1$,

$$
p_{m}(x):=\frac{x^{2 m+\nu}}{1 / a_{m}+r_{m}(x)}
$$

при всех $x \in I$, где $\nu, a_{0}, a_{1}, \ldots, a_{m}, b_{0}, b_{1}, \ldots, b_{m-1}$ - действительнъе числа, $a_{m} \neq 0$, a $r_{0}, r_{1}, \ldots, r_{m}, s_{0}, s_{1}, \ldots, s_{m-1}$ - вещественные функиии на $I$.

Обозначим через $D:=\left(d_{i} \delta_{i j}\right)_{i, j=1}^{2 m}$ диагональную матрицу с элементами

$$
d_{k}(x):=x^{-k+1 / 2}, \quad d_{k+m}(x):=x^{m+\nu-k+1 / 2}, \quad k=1, \ldots, m .
$$

В системе $(2)$ с $F=F_{2 m}$ сделаем замену $\mathbf{y}=D \mathbf{Y}$, где $\mathbf{Y}-$ новая неизвестная вектор-функция. Тогда система (2) преобразуется к виду

$$
\mathbf{Y}^{\prime}=\left(D^{-1} F_{2 m} D+D^{-1} \Lambda D-D^{-1} D^{\prime}\right) \mathbf{Y}
$$

Заметим, что

$$
\begin{gathered}
D^{-1} D^{\prime}=x^{-1} \operatorname{diag}\left(\left(-\frac{1}{2}\right),\left(-\frac{3}{2}\right), \ldots,\left(-m+\frac{1}{2}\right),\left(m+\nu-1+\frac{1}{2}\right),\right. \\
\left.\left(m+\nu-2+\frac{1}{2}\right), \ldots,\left(\nu+\frac{1}{2}\right)\right),
\end{gathered}
$$

все элементы $\widetilde{\lambda}_{i, j}, i, j=1,2, \ldots, 2 m$, матрицы $\widetilde{\Lambda}:=D^{-1} \Lambda D$ равны нулю, кроме $\tilde{\lambda}_{2 m, 1}(x)=x^{-1}(-1)^{m} \lambda / x^{\nu}$, а элементы матрицы $\widetilde{F}:=D^{-1} F_{2 m} D$ несложно выписать с помощью формул перемножения матриц, например, элементы $\widetilde{f}_{2 m-k, k+1}(x)$ имеют вид:

$$
\begin{gathered}
\tilde{f}_{2 m-k, k+1}=d_{2 m-k}^{-1} f_{2 m-k, k+1} d_{k+1}=d_{2 m-k}^{-1} \alpha_{k} d_{k+1}=(-1)^{m+1} x^{-2 k-\nu-1} p_{k}, \\
k=0,1, \ldots, m-2 .
\end{gathered}
$$

Таким же образом вычисляя другие элементы матрицы $\widetilde{F}$ с учетом условий А и В, находим, что неизвестная вектор-функция $\mathbf{Y}$ удовлетворяет системе уравнений

$$
x \frac{d \mathbf{Y}}{d x}=(A+B(x)) \mathbf{Y},
$$

где элементы $a_{i j}$ числовой матрицы $A$ и элементы $b_{i j}(x)$ матрицы-функции $B(x)$ определяются следующими равенствами:

$$
\begin{aligned}
& a_{k, k+1}=1, \quad k=1,2, \ldots, m-1, m+1, \ldots, 2 m-1 ; \\
& a_{m, m+1}=\frac{(-1)^{m}}{a_{m}}, \quad b_{m, m+1}(x)=(-1)^{m} r_{m}(x) \text {; } \\
& a_{2 m, 1}=(-1)^{m}\left(\lambda-a_{0}\right), \quad b_{2 m, 1}(x)=(-1)^{m+1} r_{0}(x), \quad \nu=0 \text {; } \\
& a_{2 m, 1}=(-1)^{m+1} a_{0}, \quad b_{2 m, 1}(x)=(-1)^{m}\left(\frac{\lambda}{x^{\nu}}-r_{0}(x)\right), \quad \nu \neq 0 \text {; }
\end{aligned}
$$




$$
\begin{gathered}
a_{2 m-k, k+1}=(-1)^{m+1} a_{k}, \quad b_{2 m-k, k+1}(x)=(-1)^{m+1} r_{k}(x), \\
k=1,2, \ldots, m-2 ; \\
a_{m+1, m}=(-1)^{m+1}\left(a_{m-1}+\frac{b_{m-1}^{2}}{a_{m}}\right), \\
b_{m+1, m}(x)=(-1)^{m+1}\left(r_{m-1}(x)+\left(\frac{1}{a_{m}}+r_{m}(x)\right)\left(b_{m-1}+s_{m-1}(x)\right)^{2}-\frac{b_{m-1}^{2}}{a_{m}}\right) ; \\
a_{k, k}=k-\frac{1}{2}, \quad a_{k+1+m, k+1+m}=k+\frac{1}{2}-m-\nu, \quad k=1,2, \ldots, m-1 ; \\
a_{m, m}=m-\frac{1}{2}-\frac{i b_{m-1}}{a_{m}}, \quad a_{m+1, m+1}=\frac{1}{2}-m-\nu-\frac{i b_{m-1}}{a_{m}} ; \\
b_{m, m}(x)=b_{m+1, m+1}(x)=-i\left(\frac{s_{m-1}(x)}{a_{m}}+b_{m-1} r_{m}(x)+s_{m-1}(x) r_{m}(x)\right) ; \\
a_{2 m-k, k}=a_{2 m+1-k, k+1}=(-1)^{m+1} i b_{k-1}, \quad k=1,2, \ldots, m-1 ; \\
b_{2 m-k, k}(x)=b_{2 m+1-k, k+1}(x)=(-1)^{m+1} i s_{k-1}(x), \quad k=1,2, \ldots, m-1 ;
\end{gathered}
$$

все неуказанные элементы в обеих матрицах $A$ и $B(x)$ равны нулю.

Пусть теперь $n=2 m+1, m=1,2 \ldots$. Предположим, что выполнено

УСловие А $(n=2 m+1)$. Функиии $p_{0}, p_{1}, \ldots, p_{m}, q_{0}, q_{1}, \ldots, q_{m}$ вещественнъе на $I,(-1)^{m} q_{m}(x)>0$ при $x \in I$ и все функции

$$
\begin{gathered}
\alpha_{m}:=p_{m}\left(2 q_{m}\right)^{-1}, \quad \alpha_{k}:=(-1)^{m} p_{k}, \quad k=0,1, \ldots, m-1, \\
\beta_{m}:=\left[(-1)^{m} 2 q_{m}\right]^{-1 / 2}(>0), \quad \beta_{m-1}:=(-1)^{m+1} q_{m-1} \beta_{m}, \\
\beta_{k}:=(-1)^{m+1} q_{k}, \quad k=0,1, \ldots, m-2,
\end{gathered}
$$

принадлежат $\mathscr{L}_{1, \mathrm{loc}}(I)$.

Определим матрицу $F_{2 m+1}:=F$, положив

$$
F_{2 m+1}=\left(\begin{array}{cccccccccccc}
0 & 1 & 0 & 0 & . & . & . & . & . & . & 0 & 0 \\
0 & 0 & 1 & 0 & . & . & . & . & . & . & 0 & 0 \\
0 & 0 & 0 & 1 & . & . & . & . & . & . & 0 & 0 \\
. & . & . & . & . & . & . & . & . & . & . & . \\
. & . & . & . & 0 & \beta_{m} & 0 & . & . & . & . & . \\
. & . & . & . & \beta_{m-1} & i \alpha_{m} & \beta_{m} & . & . & . & . & . \\
. & . & . & . & i \alpha_{m-1} & \beta_{m-1} & 0 & . & . & . & . & . \\
. & . & . & . & . & . & . & . & . & . & . & . \\
0 & \beta_{1} & i \alpha_{2} & . & . & . & . & . & . & . & 1 & 0 \\
\beta_{0} & i \alpha_{1} & \beta_{1} & . & . & . & . & . & . & . & 0 & 1 \\
i \alpha_{0} & \beta_{0} & 0 & . & . & . & . & . & . & . & 0 & 0
\end{array}\right) .
$$

Следовательно, элементы матрицы $F_{2 m+1}$ имеют следующий вид:

$$
\begin{array}{ll}
f_{2 m+1-k, k+1}=i \alpha_{k}, & k=0,1, \ldots, m, \\
f_{2 m+1-k, k}=f_{2 m+2-k, k+1}=\beta_{k-1}, & k=1,2, \ldots, m+1, \\
f_{k, k+1}=1, & k=1,2, \ldots, m-1, m+2, \ldots, 2 m,
\end{array}
$$

и $f_{i j}=0$ при всех остальных значениях $i$ и $j$. 
Выражение $l_{2 m+1} y, m \geqslant 1$, представляется обыкновенным дифференциальным выражением

$$
l_{2 m+1} y=\sum_{k=0}^{m}\left(p_{k} y^{(k)}\right)^{(k)}+i \sum_{k=0}^{m}\left[\left(q_{k} y^{(k+1)}\right)^{(k)}+\left(q_{k} y^{(k)}\right)^{(k+1)}\right],
$$

если $p_{k} \in C^{(k)}(I), k=0,1, \ldots, m ; q_{k} \in C^{(k+1)}(I), k=0,1, \ldots, m$ (см. [1; добавление A]).

В этом случае условие В имеет следующий вид.

УСловиЕ В $(n=2 m+1)$. Функции $p_{0}, p_{1}, \ldots, p_{m-1}, q_{0}, q_{1}, \ldots, q_{m-1}$ такие же, как в случае $n=2 m$, а $p_{m}, q_{m}$ представляются в виде

$$
\begin{aligned}
p_{m}(x) & :=x^{2 m+\nu}\left(a_{m}+r_{m}(x)\right), \\
q_{m}(x) & :=\frac{x^{2 m+\nu+1}}{1 / b_{m}+s_{m}(x)}
\end{aligned}
$$

при всех $x \in I$, где $a_{m}$ и $b_{m}$ - действительнье числа $и(-1)^{m} b_{m}>0$, a $r_{m} u$ $s_{m}$ - вещественные функиии на $I$.

Определим диагональные элементы $d_{k}$ матрицы $D$ в замене $\mathbf{y}=D \mathbf{Y}$ в рассматриваемом случае следующими равенствами:

$$
d_{k}:=x^{-k+1 / 2}, \quad d_{m+1+k}:=x^{\nu+m-k+1 / 2}, \quad k=1,2, \ldots, m ; \quad d_{m+1}:=x^{\nu / 2} .
$$

С помощью такой замены система (2) опять преобразуется к системе вида (4) для вектор-функции $\mathbf{Y}$, где ненулевые элементы числовой матрицы $A$ и элементы $b_{i j}(x)$ матрицы-функции $B(x)$ определяются следующими равенствами:

$$
\begin{gathered}
a_{k, k+1}=1, \quad k=1,2, \ldots, m-1, m+2, \ldots, 2 m ; \\
a_{m, m+1}=a_{m+1, m+2}=\left(\frac{1}{2} \frac{(-1)^{m}}{b_{m}}\right)^{1 / 2} ; \\
b_{m, m+1}=b_{m+1, m+2}=\left[(-1)^{m} \frac{1}{2}\left(\frac{1}{b_{m}}+s_{m}(x)\right)\right]^{1 / 2}-\left[\frac{1}{2} \frac{(-1)^{m}}{b_{m}}\right]^{1 / 2} ; \\
a_{2 m+1,1}=(-1)^{m} i\left(a_{0}-\lambda\right), \quad b_{2 m+1,1}(x)=(-1)^{m} i r_{0}(x), \quad \nu=0 ; \\
a_{2 m+1,1}=(-1)^{m} i a_{0}, \quad b_{2 m+1,1}(x)=(-1)^{m} i\left(r_{0}(x)-\frac{\lambda}{x^{\nu}}\right), \quad \nu \neq 0 ; \\
a_{2 m+1-k, k+1}=(-1)^{m} i a_{k}, \quad b_{2 m+1-k, k+1}(x)=(-1)^{m} i r_{k}(x), \quad k=1,2, \ldots, m-1 ; \\
a_{k, k}=k-\frac{1}{2}, \quad a_{m+1+k, m+1+k}=k-\nu-m-\frac{1}{2}, \quad k=1,2, \ldots, m ; \\
a_{m+1, m+1}=\frac{i a_{m}}{2 b_{m}}-\frac{\nu^{2}}{2}, \quad b_{m+1, m+1}(x)=\frac{i}{2}\left(a_{m}+r_{m}(x)\right)\left(\frac{1}{b_{m}}+s_{m}(x)\right)-\frac{i a_{m}}{2 b_{m}} \\
a_{m+1, m}=a_{m+2, m+1}=\frac{(-1)^{m}}{\sqrt{2}}\left(\frac{(-1)^{m}}{b_{m}}\right)^{1 / 2} b_{m-1} ; \\
b_{m+1, m}(x)=b_{m+2, m+1}(x)=\frac{(-1)^{m}}{\sqrt{2}}\left(\left[b_{m-1}+s_{m-1}(x)\right]\right. \\
\times\left[(-1)^{m}\left(\frac{1}{b_{m}}+s_{m}(x)\right]^{1 / 2}-\left[\frac{(-1)^{m}}{b_{m}}\right]^{1 / 2} b_{m-1}\right)
\end{gathered}
$$




$$
\begin{aligned}
a_{2 m+1-k, k} & =a_{2 m+2-k, k+1}=(-1)^{m+1} b_{k-1}, & & k=1, \ldots, m-1, \\
b_{2 m+1-k, k}(x) & =b_{2 m+2-k, k+1}(x)=(-1)^{m+1} s_{k-1}(x), & & k=1, \ldots, m-1 .
\end{aligned}
$$

В дальнейшем нам понадобится характеристический многочлен матрицы $A$. Найдем явный вид этого многочлена (для $n=2 m$ и $n=2 m+1$ ).

Пусть

$$
\operatorname{det}(A-z E)=\left\{\begin{array}{lll}
\mathfrak{F}_{n}(z, \nu), & \text { если } & \nu \neq 0 \\
\mathfrak{F}_{n}(z, 0)-\lambda, & \text { если } & \nu=0
\end{array}\right.
$$

Вычисления показывают, что

$$
\begin{aligned}
\mathfrak{F}_{2}(z, \nu)= & \frac{1}{a_{1}}\left(a_{0}+a_{1}\left[\left(z+\frac{1}{2}\right)^{2}-\left(\frac{\nu+1}{2}\right)^{2}\right]+2 i b_{0}\left(z+\frac{\nu}{2}\right)\right), \\
\mathfrak{F}_{3}(z, \nu)= & \frac{i}{2 b_{1}}\left(a_{0}+a_{1}\left[\left(z+\frac{1}{2}\right)^{2}-\left(\frac{\nu+1}{2}\right)^{2}\right]\right. \\
& \left.+2 i\left(z+\frac{\nu}{2}\right)\left(b_{0}+b_{1}\left[\left(z+\frac{1}{2}\right)^{2}-\left(\frac{\nu+1}{2}\right)^{2}\right]\right)\right) .
\end{aligned}
$$

Далее, несложные, но громоздкие вычисления с использованием метода математической индукции показывают, что при $m=1,2, \ldots$ полиномы $\mathfrak{F}_{n}(z, \nu)$ определяются следующими равенствами:

$$
\begin{aligned}
\mathfrak{F}_{2 m}(z, \nu)= & \frac{1}{a_{m}}\left(a_{0}+\sum_{k=1}^{m} a_{k} \prod_{j=0}^{k-1}\left[\left(z+\frac{\nu}{2}\right)^{2}-\left(\frac{\nu+1}{2}+j\right)^{2}\right]\right. \\
& \left.+2 i\left(z+\frac{\nu}{2}\right)\left(b_{0}+\sum_{k=1}^{m-1} b_{k} \prod_{j=0}^{k-1}\left[\left(z+\frac{\nu}{2}\right)^{2}-\left(\frac{\nu+1}{2}+j\right)^{2}\right]\right)\right), \\
\mathfrak{F}_{2 m+1}(z, \nu)= & \frac{i}{2 b_{m}}\left(a_{m} \mathfrak{F}_{2 m}(z, \nu)\right. \\
& \left.+2 i\left(z+\frac{\nu}{2}\right) b_{m} \prod_{j=0}^{m-1}\left[\left(z+\frac{\nu}{2}\right)^{2}-\left(\frac{\nu+1}{2}+j\right)^{2}\right]\right) .
\end{aligned}
$$

\section{§ 2. Случай аналитических коэффициентов}

В этом параграфе сначала рассмотрим случай, когда $I:=(0,1]$. Пусть $\nu \leqslant 0$, $\nu$ - целое число. Предположим, что коэффициенты $p_{0}, p_{1}, \ldots p_{m}, q_{0}, q_{1}, \ldots, q_{m}$ квазидифференциального выражения $l_{n}$, порожденного матрицей $F_{n}$, удовлетворяют условию А и, кроме того, выполняется

УСловие $\mathrm{C}(I=(0,1])$. Функиии $r_{k}(z)$ и $s_{k}(z)$ из условия В представляются в виде сходящихся при $|z| \leqslant x_{0}(\leqslant 1)$ степенных рядов

$$
\begin{array}{rlrlrl}
r_{k}(z) & :=\sum_{j=1}^{+\infty} a_{j}^{(k)} z^{j}, & & k=0,1, \ldots, m, & \text { если } \quad n=2 m \quad \text { или } n=2 m+1 ; \\
s_{k}(z):=\sum_{j=1}^{+\infty} b_{j}^{(k)} z^{j}, & k=0,1, \ldots, m-1, & \text { если } & n=2 m, \\
& k=0,1, \ldots, m, & \text { если } & n=2 m+1 .
\end{array}
$$


Справедлива следующая теорема.

Теорема 3. Пусть элементы матрицы $F_{n}$ удовлетворяют условиям А

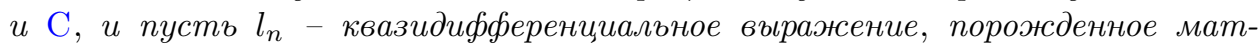
рищей $F_{n}$. Тогда максимальное число линейно независимых решений уравнения (1), принадлежащих пространству $\mathscr{L}_{2}(I)$, равно

1) при $\nu<0$ числу корней полинома $\mathfrak{F}_{n}(z, \nu)$, лежащих в области $\operatorname{Re} z>0$, и не зависит от $\lambda$;

2) при $\nu=0$ числу корней полинома $\mathfrak{F}_{n}(z, 0)-\lambda$, лежащих в области $\operatorname{Re} z>0$, и если $n=2 m$, то $n_{+}=n_{-}=m$, если же $n=2 m+1$, mо $n_{+}=m$, $n_{-}=m+1$.

При этом в случае $\nu<0$ спектр любого самосопряюенного расширения оператора $L_{0}$ дискретный.

ДокАЗАТЕльство. Из списков формул, приведенных выше в 11 для элементов матрицы-функции $B(x)$, и условия $\mathrm{C}$ как в случае $n=2 m$, так и в случае $n=2 m+1$ непосредственно следует, что эта матрица-функция представляется сходящимся при $|z| \leqslant x_{0}(\leqslant 1)$ степенным рядом

$$
B(z)=\sum_{j=1}^{\infty} z^{j} B_{j},
$$

где $B_{j}$ - постоянные матрицы. Следовательно, система дифференциальных уравнений (4) в комплексной области с коэффициентами, удовлетворяющими условиям А и С, является системой с единственной регулярной особой точкой при $x=0$. Поэтому, применяя соответствующие результаты для решений систем дифференциальных уравнений такого типа (см. [12; гл. XV, §11, замечание 2 к теореме 2]), получаем, что фундаментальная матрица решений системы (4) имеет вид

$$
\Phi(x)=P(x)\left(\begin{array}{cccc}
x^{z_{1}} & 0 & \ldots & 0 \\
0 & x^{z_{2}} & \ldots & 0 \\
\ldots \ldots & \ldots & \ldots & \ldots \\
0 & 0 & \ldots & x^{z_{n}}
\end{array}\right)\left(\begin{array}{cccc}
1 & q_{12} & \ldots & q_{1 n} \\
0 & 1 & \ldots & q_{2 n} \\
\ldots & \ldots & \ldots & \ldots \\
0 & 0 & \ldots & 1
\end{array}\right)
$$

где $P(x)=\sum_{m=0}^{\infty} x^{m} P_{m}-$ сходящийся при $|x| \leqslant x_{0}(<1)$ степенной ряд, матрица $P_{0}=C, \operatorname{det}(C) \neq 0, C$ - матрица преобразования матрицы $A$ к жордановой форме; если матрица $A$ имеет жорданову форму, то $C=E$. Числа $z_{1}, z_{2}, \ldots, z_{n}$ - характеристические числа матрицы $A$, т.е. корни уравнения $\mathfrak{F}_{n}(z, \nu)=0$, если $\nu<0$, и $\mathfrak{F}_{n}(z, 0)-\lambda=0$, если $\nu=0$, расположенные в порядке $\operatorname{Re} z_{1} \geqslant \operatorname{Re} z_{2} \geqslant \cdots \geqslant \operatorname{Re} z_{n} ;$ a $q_{i j}, i, j=1,2, \ldots, n, i<j,-$ многочлены от $\ln x$ степени не выше $g-1$, где $g$ - максимальное количество характеристических чисел $z_{i}$, отличающихся между собой на целое число.

Далее, используя представление (5) и метод Фробениуса (см., например, [13; гл. IV, §8], [14; гл. XVI]), можно показать, что уравнение (1) имеет фундаментальную систему решений такую, что для каждого решения из этой системы справедливо соотношение

$$
y(x)=x^{z-1 / 2}(\ln x)^{s}(1+o(1)), \quad x \rightarrow+0 .
$$


Другое доказательство последнего утверждения мы приведем в следующем параграфе (см. доказательство теоремы 5), поэтому здесь подробности опускаем.

Функции вида (6) принадлежат пространству $\mathscr{L}_{2}(0,1]$ тогда и только тогда, когда

$$
\int_{0}^{x_{0}}\left|x^{2 z-1}(\ln x)^{2 s}\right| d x<\infty,
$$

а это выполняется тогда и только тогда, когда $\operatorname{Re} z>0$.

Далее, для доказательства п. 2) заметим, что для полинома $\mathfrak{F}_{n}(z, 0)-\lambda$ справедливо равенство

$$
\overline{\mathfrak{F}_{n}(\overline{-z}, 0)-\lambda}=\mathfrak{F}_{n}(z, 0)-\bar{\lambda}
$$

Отсюда для дефектных чисел легко вывести неравенство $n_{+}+n_{-} \leqslant n$. Учитывая оценки для $n_{+}$и $n_{-}$(см. теорему 1$)$, получаем, что если $n=2 m$, то $n_{+}=n_{-}=m$, если же $n=2 m+1$, то $n_{+}=m, n_{-}=m+1$.

Таким образом, доказаны пп. 1) и 2).

Как мы уже отмечали во введении, дефектное число $n_{+}\left(n_{-}\right)$оператора $L_{0}$ равно максимальному числу линейно независимых решений уравнения (1) при $\operatorname{Im} \lambda>0(\operatorname{Im} \lambda<0)$, принадлежащих пространству $\mathscr{L}_{2}(I)$. Таким образом, в случае $\nu<0$ ( $\nu$ - целое число) согласно п. 1$)$ дефектные числа оператора $L_{0}$ равны и, следовательно, оператор $L_{0}$ имеет самосопряженные расширения. Используя равенства (6) и (5), можно показать, что функция Грина любого самосопряженного расширения оператора $L_{0}$ является ядром Гильберта-Шмидта и мероморфной функцией от $\lambda$. Из этого следует дискретность спектра любого самосопряженного расширения. Теорема 3 доказана.

Пусть теперь $I:=[1,+\infty), \nu \geqslant 0, \nu$ - целое число. Предположим, что коэффициенты $p_{0}, p_{1}, \ldots, p_{m}, q_{0}, q_{1}, \ldots, q_{m}$ квазидифференциального выражения $l_{n}$, порожденного матрицей $F_{n}$, удовлетворяют условию А для этого случая (см. §1), при этом условие С имеет вид:

УСловие $\mathrm{C}(I=[1,+\infty))$. Функиии $r_{k}(z)$ u $s_{k}(z)$ из условия В представляются в виде сходящихся при $|z| \geqslant x_{0}(\geqslant 1)$ степенных рядов

$$
\begin{aligned}
& r_{k}(z):=\sum_{j=1}^{+\infty} a_{j}^{(k)} z^{-j}, \quad k=0,1, \ldots, m, \quad \text { если } \quad n=2 m \quad \text { или } \quad n=2 m+1
\end{aligned}
$$

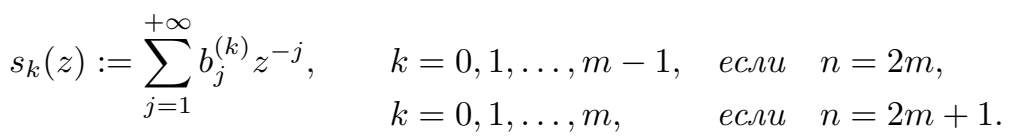

\section{Справедлива следующая}

ТЕОрема 4. Пусть элементы матрицы $F_{n}$ удовлетворяют условиям А u C, $и$ пусть $l_{n}$ - квазидифференциальное выражение, порожденное матрищей $F_{n}$. Тогда максимальное число линейно независимых решений уравнения (1), принадлежащих пространству $\mathscr{L}_{2}(I)$, равно

1) при $\nu>0$ числу корней полинома $\mathfrak{F}_{n}(z, \nu)$, лежащих в области $\operatorname{Re} z<0$, и не зависит от $\lambda$; 
2) при $\nu=0$ числу корней полинома $\mathfrak{F}_{n}(z, 0)-\lambda$, лежащих в области $\operatorname{Re} z<0$, и если $n=2 m$, mo $n_{+}=n_{-}=m$, если $n=2 m+1$, mo $n_{+}=m, n_{-}=m+1^{1}$. При этом в случае $\nu>0$ спектр любого самосопряженного расширения опеpатора $L_{0}$ дискретный.

ДокАЗАТЕЛЬство. Легко проверить, что если выполняются условия А и С, то в данном случае система (4) является системой с регулярной особой точкой в $+\infty$. Далее, с помощью стандартной замены $x=1 / \xi$ доказательство справедливости теоремы 4 сводится к теореме 3.

ЗАмечАниЕ. Отметим, что теорема 2 является частным случаем теоремы 4. Мы предполагаем, что С.А. Орлов доказывал эту теорему именно способом, изложенным нами выше, однако подробные публикации нам неизвестны.

\section{§ 3. Случай неаналитических коэффициентов}

В этом параграфе мы будем опираться на следующую лемму.

Лемма 1 (см. [13; гл. III, задача 35]). Рассмотрим систему дифберенииальных уравнений

$$
U^{\prime}=A U+R(t) U
$$

где матрица $A$ постоянна, жорданова форма матрицы А имеет жордановъ клетки $J_{k}, k \geqslant 1, u$ максимальное число строк для всех клеток $J_{k}, k \geqslant 1$, равно $r+1$. Предположим, что

$$
\int_{1}^{\infty} t^{r}\|R(t)\| d t<\infty .
$$

Пусть z - характеристический корень $A$, и пусть уравнение $y^{\prime}=$ Ау имеет решения вида

$$
e^{z t} t^{k} c+O\left(e^{z t} t^{k-1}\right)
$$

где с - постоянный вектор. Тогда уравнение (7) имеет решение $\varphi$ такое, что

$$
\varphi(t)=e^{z t} t^{k}(c+o(1)), \quad t \rightarrow+\infty .
$$

Рассмотрим сначала случай $I:=[1,+\infty), \nu \geqslant 0$ (необязательно целое) и преобразуем систему дифференциальных уравнений (4), полагая $x=e^{t}$. Тогда система (4) преобразуется к виду (7), где $U(t)=\mathbf{Y}\left(e^{t}\right)$, а $R(t)=B\left(e^{t}\right)$.

Пусть $z$ - характеристический корень матрицы $A$ кратности $r_{0}$. Первая координата системы дифференциальных уравнений $U^{\prime}(t)=A U(t)$ удовлетворяет скалярному дифференциальному уравнению $n$-го порядка с постоянными коэффициентами, поэтому эта система имеет решения вида

$$
e^{z t} t^{k} c+O\left(e^{z t} t^{k-1}\right), \quad k=0,1, \ldots, r_{0}-1,
$$

$c$ - постоянный вектор.

Кроме того, в рассматриваемом случае система (7) также построена по одному скалярному дифференциальному уравнению. Легко заметить, что поэтому

\footnotetext{
1Эта часть утверждения в формулировке теоремы 2 работы [11] содержит ошибку, исправленную здесь.
} 
каждому характеристическому корню матрицы $A$ будет соответствовать только одна клетка в ее жордановой форме. Следовательно, если максимальная размерность жордановой клетки, как и в лемме 1 , равна $r+1$, то $r+1$ - это кратность характеристического корня матрицы $A$ наибольшей кратности.

Пусть для матрицы $R(t)$ выполняется условие (7), тогда, применяя лемму 1 , находим, что эта система имеет решения вида

$$
\varphi(t)=e^{z t} t^{k}(c+o(1)), \quad t \rightarrow+\infty, \quad k=0,1, \ldots, r_{0}-1, \quad r_{0} \leqslant r+1 .
$$

Возвращаясь к переменной $x$ и учитывая преобразование $\mathbf{y}=D \mathbf{Y}$, получаем, что уравнение (1) имеет фундаментальную систему решений, состоящую из функций вида

$$
y(x)=c x^{z-1 / 2}(\ln x)^{k}(1+o(1)), \quad x \rightarrow+\infty, \quad k=0,1, \ldots, r_{0}-1, \quad r_{0} \leqslant r+1,
$$

где $z$ - характеристический корень матрицы $A, c$ - ненулевое число.

Таким образом, в силу условия $(7)$, если матрица $B(x)$ такова, что

$$
\int_{x_{0}}^{\infty} \frac{(\ln x)^{r}}{x}\|B(x)\| d x<\infty,
$$

где $x_{0}=e^{t_{0}}$, то система дифференциальных уравнений (4) имеет фундаментальную систему решений, состоящую из функций вида (9).

Легко заметить, что последнее условие в терминах функций $r_{k}(x)$ и $s_{k}(x)$ переформулируется следующим образом.

Условие D $(I=[1,+\infty))$. Если $n=2 m, m o$

$$
\begin{array}{ll}
\int_{x_{0}}^{\infty}\left|r_{k}(x)\right| \frac{(\ln x)^{r}}{x} d x<+\infty, & k=0,1, \ldots, m, \\
\int_{x_{0}}^{\infty}\left|s_{k}(x)\right| \frac{(\ln x)^{r}}{x} d x<+\infty, & k=0,1, \ldots, m-1,
\end{array}
$$

и функиия $s_{m-1}(x)$ ограничена при $x \geqslant x_{0}(\geqslant 1)$.

Если $n=2 m+1$, то функции $r_{0}, r_{1}, \ldots, r_{m}, s_{0}, s_{1}, \ldots, s_{m-1}$ такие же, как и в случае $n=2 m$, а функиия $s_{m}(x)$ ограничена при $x \geqslant x_{0}(\geqslant 1)$ u

$$
\int_{x_{0}}^{\infty}\left|s_{m}(x)\right| \frac{(\ln x)^{r}}{x} d x<+\infty .
$$

Итак, мы полностью доказали следующую теорему.

ТеОРема 5. Пусть $\nu \geqslant 0$ и элементы матрицы $F_{n}$ удовлетворяют условиям А и D в этом случае. Предположим, что $z_{1}, z_{2}, \ldots, z_{q}, z_{q+1}, \ldots, z_{q+j}-$ все различные характеристические корни матричы $A$, причем $z_{1}, z_{2}, \ldots, z_{q}$-однократные корни, при $1 \leqslant p \leqslant j$ кратность корня $z_{q+p}$ равна $r_{p} u \sum_{p=1}^{j} r_{p}+q=n$, $\max _{1 \leqslant p \leqslant j} r_{p}=r+1$. Тогда уравнение (1) имеет фундаментальную систему решений $y_{k}(x)$ такую, что при $x \rightarrow+\infty$

$$
\begin{array}{rlrl}
y_{k}(x) & =c x^{z_{k}-1 / 2}(1+o(1)), & k & =1,2, \ldots, q, \\
y_{k+i}(x) & =c x^{z_{q+p}-1 / 2}(\ln x)^{i}(1+o(1)), & k & =q+r_{1}+\cdots+r_{p-1}, \\
i & =0,1, \ldots, r_{p}-1 .
\end{array}
$$


Далее, рассуждая так же, как и в конце доказательства теоремы $3 \S 2$, получаем результаты о дефектных числах оператора $L_{0}$ и характере его спектра, т.е. справедлива

ТеОрема 6. Пусть выполняются условия теоремы 5. Тогда справедливы все утверждения теоремы 4.

Пусть теперь $I:=(0,1], \nu \leqslant 0$ (необязательно целое). Проводя рассуждения, аналогичные предыдущему случаю, и производя в системе (4) замену $x=e^{-t}$ $(x \rightarrow+0$ при $t \rightarrow+\infty)$, получаем, что уравнение (1) имеет фундаментальную систему решений, состоящую из функций вида

$y(x)=c x^{z-1 / 2}(-\ln x)^{k}(1+o(1)), \quad x \rightarrow+0, \quad k=0,1, \ldots, r_{0}-1, \quad r_{0} \leqslant r+1$,

где $z$ - характеристический корень матрицы $A, c$ - ненулевое число.

Условие D на коэффициенты дифференциального уравнения (1) в данном случае имеет следующий вид:

УСловие D $(I=(0,1])$. Если $n=2 m, m o$

$$
\begin{array}{ll}
\int_{0}^{x_{0}}\left|r_{k}(x)\right| \frac{|\ln x|^{r}}{x} d x<+\infty, & k=0,1, \ldots, m, \\
\int_{0}^{x_{0}}\left|s_{k}(x)\right| \frac{|\ln x|^{r}}{x} d x<+\infty, & k=0,1, \ldots, m-1,
\end{array}
$$

и функция $s_{m-1}(x)$ ограничена при $0<x \leqslant x_{0}(\leqslant 1)$.

Если $n=2 m+1$, то функции $r_{0}, r_{1}, \ldots, r_{m}, s_{0}, s_{1}, \ldots, s_{m-1}$ такие же, как и в случае $n=2 m$, а функиия $s_{m}(x)$ ограничена при $0<x \leqslant x_{0}(\leqslant 1) u$

$$
\int_{0}^{x_{0}}\left|s_{m}(x)\right| \frac{|\ln x|^{r}}{x} d x<+\infty .
$$

Таким образом, справедлива

ТеОРема 7. Пусть $\nu \leqslant 0$ и элементы матрицы $F_{n}$ удовлетворяют условиям А $и$ D в этом случае. Предположим, что $z_{1}, z_{2}, \ldots, z_{q}, z_{q+1}, \ldots, z_{q+j}-$ все различные характеристические корни матрицы $A$, причем $z_{1}, z_{2}, \ldots, z_{q}$-однократные корни, при $1 \leqslant p \leqslant j$ кратность корня $z_{q+p}$ равна $r_{p} u \sum_{p=1}^{j} r_{p}+q=n$, $\max _{1 \leqslant p \leqslant j} r_{p}=r+1$. Тогда уравнение (1) имеет фундаментальную систему решений $y_{k}(x)$ такую, что при $x \rightarrow+0$

$$
\begin{array}{rlrl}
y_{k}(x) & =c x^{z_{k}-1 / 2}(1+o(1)), & k & =1,2, \ldots, q, \\
y_{k+i}(x) & =c x^{z_{q+p}-1 / 2}(-\ln x)^{i}(1+o(1)), & k & =q+r_{1}+\cdots+r_{p-1}, \\
& i & =0,1, \ldots, r_{p}-1 .
\end{array}
$$

Применяя теорему 7, легко доказать, что справедливо

СлеДСтвиЕ 1. Пусть $l_{2 m}[y]=\left(x^{p} a(x) y^{(m)}\right)^{(m)}$, где $p \in[1,2 m), a(x)-$ интегрируемая вещественная функиия на отрезке $[0,1] u|a(x)| \geqslant m>0$ для всех $x \in[0,1]$. Тогда 
1) если $p$ - не челое число и функция $а(x)$ удовлетворяет условию

$$
\int_{0}^{1} \frac{|a(x)-a|}{x} d x<\infty
$$

где а - некоторое ненулевое число, то уравнение $l_{2 m} y=\lambda$ имеет фундаментальную систему решений $y_{k}(x)$ такую, что при $x \rightarrow+0$

$$
y_{k}= \begin{cases}x^{k-1}(1+o(1)), & k=1,2, \ldots, m \\ x^{k-p-1}(1+o(1)), & k=m+1, m+2, \ldots, 2 m\end{cases}
$$

2) если же $p$ - целое число, а функция $а(x)$ удовлетворяет условию

$$
\int_{0}^{1} \frac{|a(x)-a|}{x}|\ln x| d x<\infty
$$

где а - некоторое ненулевое число, то уравнение $l_{2 m} y=\lambda$ у имеет фундаментальную систему решений $y_{k}(x)$ такую, что при $p \in[1, m] u x \rightarrow+0$

$$
y_{k}= \begin{cases}x^{k-1}(1+o(1)), & k=1,2, \ldots, m \\ x^{k-p-1} \ln x(1+o(1)), & k=m+1, m+2, \ldots, m+p \\ x^{k-p-1}(1+o(1)), & k=m+p+1, m+p+2, \ldots, 2 m,\end{cases}
$$

a при $p \in[m+1,2 m-1] u x \rightarrow+0$

$$
y_{k}= \begin{cases}x^{k-1}(1+o(1)), & k=1,2, \ldots, m ; \\ x^{k-p-1}(1+o(1)), & k=m+1, m+2, \ldots, p ; \\ x^{k-p-1} \ln x(1+o(1)), & k=p+1, p+2, \ldots, 2 m .\end{cases}
$$

Рассуждая так же, как и при доказательстве теоремы 3, мы приходим к следующей теореме.

ТЕОРема 8. Пусть выполняются условия теоремы 7. Тогда справедливы все утверждения теоремы 3.

Очевидным следствием теоремы 8 является известный результат:

СлеДСтвиЕ 2. Пусть дифференциальное выражение $l_{2 m}[y]$ такое же, как и в следствии 1. Тогда дефектное число $n_{+}$оператора $L_{0}$ определяется по формуле

$$
n_{+}= \begin{cases}2 m, & \text { если } p \in\left[1, m+\frac{1}{2}\right) \\ 3 m-\left[p+\frac{1}{2}\right], & \text { если } p \in\left[m+\frac{1}{2}, 2 m\right) .\end{cases}
$$

Применяя результаты следствий 1 и 2, можно определить индекс дефекта некоторых дифференциальных операторов с двумя сингулярными концами; в частности, получить новые доказательства теорем о дефектных числах операторов типа Лежандра (см. [9; гл. 7]). 


\section{§ 4. Индекс дефекта одночленного иррегулярного дифференциального оператора четного порядка}

Рассмотрим обыкновенное дифференциальное выражение произвольного порядка $2 m, m=1,2, \ldots$,

$$
l_{2 m}[y](x)=\left(x^{p} a(x) y^{(m)}\right)^{(m)}(x), \quad x \in(-1,1),
$$

где $p \in\{1, \ldots, 2 m-1\}$, а вещественная достаточное число раз дифференцируемая функция $a(x)$ отлична от нуля при всех $x \in[-1,1]$.

Пусть $L_{0}^{(p)}$ - минимальный замкнутый симметрический оператор, порожденный дифференциальным выражением $l_{2 m}$ в гильбертовом пространстве $\mathscr{L}_{2}(I)$, а $n_{+}^{(p)}\left(n_{-}^{(p)}\right)$ - дефектные числа оператора $L_{0}^{(p)}$ в верхней (нижней) открытой комплексной полуплоскости. Очевидно, что $n_{+}^{(p)}=n_{-}^{(p)}$, обозначим символом $n^{(p)}$ их общее значение.

Данный параграф посвящен определению числа $n^{(p)}$, а именно, здесь мы доказываем, что справедлива следующая

Теорема 9. При $p=1,2, \ldots, 2 m-1$ справедлива формула

$$
n^{(p)}= \begin{cases}2 m+p, & \text { если } p=1,2, \ldots, m ; \\ 4 m-p, & \text { если } p=m+1, m+2, \ldots, 2 m-1 .\end{cases}
$$

Перечислим основные понятия и факты, используемые в доказательстве теоремы 9 .

Согласно общему определению (см. введение) квазипроизводные заданной функции $f(x) \in C^{\infty}(-1,1)$ для выражения $l_{2 m}$ определяются по формуле

$$
f^{[l]}(x)= \begin{cases}f^{(l)}(x), & \text { если } l=0,1, \ldots, m-1 ; \\ \left(x^{p} a(x) f^{(m)}\right)^{(l-m)}(x), & \text { если } l=m, m+1, \ldots, 2 m-1 .\end{cases}
$$

Несложно доказать, что квазипроизводные любой функции $f(x) \in C_{0}^{\infty}(-1,1)$ обладают следующими свойствами:

- в случае $p \in\{1,2, \ldots, m\}$

1) $f^{[l]}(0)=0$ и $f^{[l]}(x)=O\left(x^{p+m-l}\right)$ при $x \rightarrow 0$, если $l=m, m+1, \ldots$, $p+m-1$

2 ) для произвольных $2 m-p$ комплексных чисел $b_{l}$ при $l=0,1, \ldots, m-1$ и $l=m+p, m+p+1, \ldots, 2 m-1$ можно найти функцию $f(x) \in$ $C_{0}^{\infty}(-1,1)$ такую, что $f^{[l]}(0)=b_{l}$ для указанных значений индекса $l$;

- в случае $p \in\{m+1, m+2, \ldots, 2 m-1\}$

$\left.1^{\prime}\right) f^{[l]}(0)=0$ и $f^{[l]}(x)=O\left(x^{p+m-l}\right)$ при $x \rightarrow 0$, если $l=m, m+1, \ldots$, $2 m-1$;

$2^{\prime}$ ) для произвольных $m$ комплексных чисел $b_{l}$ при $l=0,1, \ldots, m-1$ можно найти функцию $f(x) \in C_{0}^{\infty}(-1,1)$ такую, что $f^{[l]}(0)=b_{l}$ для указанных значений индекса $l$.

Рассмотрим два вспомогательных дифференциальных выражения

$$
\begin{aligned}
& l_{2 m,-}[f](x)=(-1)^{m}\left(x^{p} a(x) f^{(m)}\right)^{(m)}(x), \quad x \in(-1,0), \\
& l_{2 m,+}[f](x)=(-1)^{m}\left(x^{p} a(x) f^{(m)}\right)^{(m)}(x), \quad x \in(0,1) \text {. }
\end{aligned}
$$


Зафиксируем комплексное число $\lambda, \operatorname{Im} \lambda \neq 0$, и рассмотрим дифференциальные уравнения

$$
\begin{aligned}
& l_{2 m,+}[y](x)=\lambda y(x), \quad x \in(0,1), \\
& l_{2 m,-}[y](x)=\lambda y(x), \quad x \in(-1,0) \text {. }
\end{aligned}
$$

Обозначим символом $N_{p}$ дефектное подпространство симметрического оператора $L_{0}^{(p)}$, отвечающее числу $\bar{\lambda}$, а $N_{p,-}$ и $N_{p,+}$ - дефектные подпространства вспомогательных симметрических минимальных операторов $L_{0,-}^{(p)}$ и $L_{0,+}^{(p)}$, порожденных дифференциальными выражениями $l_{2 m,-}[f](x)$ в $\mathscr{L}_{2}(-1,0)$ и $l_{2 m,+}[f](x)$ в $\mathscr{L}_{2}(0,1)$ соответственно, отвечающие указанному числу $\bar{\lambda}$. Дефектные числа операторов, порожденных выражениями $l_{2 m,-}$ и $l_{2 m,+},-$ $\operatorname{dim} N_{p,-}$ и $\operatorname{dim} N_{p,+}$ - определяются максимальным числом линейно независимых решений уравнений (12) и (11), принадлежащих пространству $\mathscr{L}_{2}(-1,0)$ и $\mathscr{L}_{2}(0,1)$ соответственно, и формулы для их вычисления приведены в следствии 2 .

Для $x \in(-1,0) \cup(0,1)$ определим билинейную форму $[f, g](x)$ для любых двух функций $f$ и $g$, имеющих абсолютно непрерывные производные всех порядков до $(2 m-1)$-го порядка включительно в некоторой окрестности точки $x$, формулой

$$
[f, g](x)=\sum_{j=1}^{2 m}(-1)^{j+1} f^{[2 m-j]}(x) \bar{g}^{[j-1]}(x) .
$$

Отметим, что эта билинейная форма возникает в известном тождестве Лагранжа для дифференциального выражения $l_{2 m}$.

Символами $[f, g](-0)$ и $[f, g](+0)$ обозначим

$$
[f, g](-0)=\lim _{x \rightarrow-0}[f, g](x), \quad[f, g](+0)=\lim _{x \rightarrow+0}[f, g](x),
$$

если указанные пределы существуют.

Справедлива следующая

ЛЕмма 2. Пусть $f(x)$ - бесконечно дифферениируемая функиия на $[-1,1]$. Тогда

1) при $p \in\{1,2, \ldots, m\}$ уравнение (11) имеет фундаментальную систему решений $y_{k}, k=1,2, \ldots, 2 m$, все элементы которой принадлежат пространству $\mathscr{L}_{2}(0,1)$ и для которых значения $\left[f, y_{k}\right](+0)$ вычисляются по формуле

$$
\left[f, y_{k}\right](+0)= \begin{cases}\alpha_{k} f^{[2 m-k]}(0), & \text { если } k=1,2, \ldots, m-p \\ 0, & \text { если } k=m-p+1, m-p+2, \ldots, m \\ \alpha_{k} f^{[2 m-k]}(0), & \text { если } k=m+1, m+2, \ldots, 2 m ;\end{cases}
$$

2) при $p \in\{m+1, m+2, \ldots, 2 m-1\}$ уравнение (11) имеет фундаментальную систему решений $y_{k}, k=1,2, \ldots, 2 m, 3 m-p$ элементов $y_{1}, y_{2}, \ldots, y_{m}$, $y_{p+1}, y_{p+2}, \ldots, y_{2 m}$ которой принадлежат пространству $\mathscr{L}_{2}(0,1)$ и для которых значения $\left[f, y_{k}\right](+0)$ вычисляются по формулам

$$
\left[f, y_{k}\right](+0)= \begin{cases}\beta_{k} f^{[2 m-k]}(0), & \text { если } k=p+1, p+2, \ldots, 2 m ; \\ 0 & \text { во всех остальных случалх. }\end{cases}
$$


Здесъ $\alpha_{k}, k \in\{1,2, \ldots, 2 m\} \backslash\{m-p+1, m-p+2, \ldots, m\}, u \beta_{k}, k \in$ $\{p+1, p+2, \ldots, 2 m\},-$ ненулевые постоянные.

ДокАЗАТЕЛьство. Предварительные сведения о структуре базиса пространства $N_{p,+}$ содержатся в следствии 1 . Для вычисления указанных в лемме 2 пределов $\left[f, y_{k}\right](+0)$ требуется уточнение асимптотических формул, приведенных в этом следствии. Если дополнительно предположить, что функция $a(x)$ представляется сходящимся степенным рядом в окрестности нуля, то в формулах (5) содержится полная информация о фундаментальной системе решений $y_{k}, k=1,2, \ldots, 2 m$, в том числе и о базисе пространства $N_{p,+}$. Анализ формул (5) и свойства 1$\left.), 2), 1^{\prime}\right), 2^{\prime}$ ) квазипроизводных функций $f$, перечисленные выше, показывают, что в пределах $[f, y(\cdot, \lambda)](+0)$ параметр $\lambda$ отсутствует. Если же $a(x)$ является только достаточное число раз дифференцируемой функцией, то справедливость последнего утверждения следует из метода последовательных приближений, используемого при доказательстве леммы 1 и этих же свойств квазипроизводных. Из вышесказанного вытекает, что указанные пределы равны пределам $[f, y(\cdot, 0)](+0)$. Теперь доказательство леммы 2 можно завершить, если для вычисления искомых пределов воспользоваться формулой

$y(x)=c_{0}+c_{1} x+\cdots+c_{m-1} x^{m-1}+\int_{x}^{1} \frac{(x-t)^{m-1}}{(m-1) !} \frac{c_{m}+c_{m+1} t+\cdots+c_{2 m-1} t^{m-1}}{t^{p} a(t)} d t$

общего решения уравнения (11) при $\lambda=0$.

Отметим, что заменой $x \mapsto-x$ уравнение (12) сводится к уравнению вида (11), поэтому в пространстве $N_{p,-}$ существует базис $\widetilde{y}_{k}(x)$, элементы которого обладают свойствами, перечисленными в лемме 2.

Символами $y_{+}(x)$ и $y_{-}(x)$ обозначим сужение функции $y(x)$, заданной при $x \in[-1,1]$ на $[0,1]$ и $[-1,0]$ соответственно.

Справедлива следующая

ЛЕмма 3 (см. [10]). При любом натуральном р дефектное подпространство $N_{p}$ оператора $L_{0}^{(p)}$ совпадает с линейным многообразием, состоящим из функций $y(x) \in \mathscr{L}_{2}(-1,1)$, обладающих тремя свойствами:

1) $y_{-}(x) \in N_{p,-}, y_{+}(x) \in N_{p,+}$;

2) для любой функции $f(x) \in C_{0}^{\infty}(-1,1)$ существуют односторонние предельь $[f, y](-0) u[f, y](+0)$;

3) в точке $x=0$ для любой $f(x) \in C_{0}^{\infty}(-1,1)$ выполняется условие сопряжения $[f, y](-0)=[f, y](+0)$.

ДокАЗАТЕЛЬСтво тЕОРЕмы 9. Схема доказательства этой теоремы одинакова как для случая $p=1,2, \ldots, m$, так и для случая $p=m+1, m+2, \ldots$, $2 m-1$ и повторяет рассуждения, приведенные в работе [10], для первого случая. Итак, пусть $p=m+1, m+2, \ldots, 2 m-1$. Множество функций из $\mathscr{L}_{2}(-1,1)$, обладающих свойством 1 ) леммы 3 , есть совокупность функций $y(x)$, заданных 
на интервале $(-1,1)$, для которых справедливы представления

$$
\begin{array}{ll}
y_{-}(x)=\sum_{k=1}^{m} d_{k} \widetilde{y}_{k}(x)+\sum_{k=p+1}^{2 m} d_{k} \widetilde{y}_{k}(x), & \\
y_{+}(x)=\sum_{k=1}^{m} c_{k} y_{k}(x)+\sum_{k=p+1}^{2 m} c_{k} y_{k}(x), \quad & x \in(0,1),
\end{array}
$$

с произвольными комплексными коэффициентами $c_{k}, d_{k}, k=1,2, \ldots, m, p+1$, $p+2, \ldots, 2 m$, где $\widetilde{y}_{k}(x)$ и $y_{k}(x)$ - базисы пространств $N_{p,-}$ и $N_{p,+}$, обладающие свойствами, перечисленными в лемме 2.

В силу леммы 2, формулы (13) и линейности формы $[f, g](x)$ получаем, что для каждой функции $y(x)$ и любой функции $f(x) \in C_{0}^{\infty}(-1,1)$ справедливы равенства

$$
\begin{aligned}
& {\left[f, y_{-}\right](-0)=\sum_{k=p+1}^{2 m} d_{k} \widetilde{\beta}_{k} f^{[2 m-k]}(0),} \\
& {\left[f, y_{+}\right](+0)=\sum_{k=p+1}^{2 m} c_{k} \beta_{k} f^{[2 m-k]}(0),}
\end{aligned}
$$

где $\beta_{k}$ и $\widetilde{\beta}_{k}-$ ненулевые числа.

Выделим из множества функций $y(x)$ совокупность таких, которые дополнительно обладают свойством 3 ) леммы 3 . Выполнение этого условия для функции $y(x)$ с произвольной функцией $f(x) \in C_{0}^{\infty}(-1,1)$ в силу свойства $\left.2^{\prime}\right)$ для квазипроизводных равносильно системе $2 m-p$ ограничений

$$
\beta_{k} c_{k}=\widetilde{\beta}_{k} d_{k}, \quad k=p+1, p+2, \ldots, 2 m,
$$

на $6 m-2 p$ коэффициентов $c_{k}, d_{k}, k=1,2, \ldots, m, p+1, p+2, \ldots, 2 m$, присутствующих в представлениях (13).

Таким образом, по лемме 3 дефектное подпространство $N_{p}$ оператора $L_{0}^{(p)}$ есть линейное многообразие функций $y(x)$, допускающих представление $(13)$ с коэффициентами $c_{k}, d_{k}, k=1,2, \ldots, m, p+1, p+2, \ldots, 2 m$, которые связаны соотношениями (14), а в остальном произвольны. Размерность этого подпространства равна количеству тех из перечисленных $6 m-2 p$ коэффициентов, которые при этих условиях могут принимать произвольные значения.

В соотношении (14)

- отсутствуют коэффициенты $c_{k}, d_{k}$ при $k=1,2, \ldots, m$, следовательно, эти $2 m$ коэффициентов произвольны;

- среди коэффициентов $c_{k}, d_{k}$ при $k=p+1, p+2, \ldots, 2 m$ произвольны только $2 m-p$ коэффициентов.

Следовательно, общее число рассматриваемых коэффициентов, принимающих произвольные значения, равно $2 m+2 m-p=4 m-p$, а значит, в данном случае дефектное число $n^{(p)}$ оператора $L_{0}^{(p)}$ определяется по формуле $n^{(p)}=4 m-p$.

Таким образом, теорема 9 доказана. 


\section{Список литературы}

[1] W. N. Everitt, L. Marcus, Boundary value problems and symplectic algebra for ordinary differential and quasi-differetial operators, Math. Surveys Monogr., 61, Amer. Math. Soc., Providence, RI, 1999.

[2] М. А. Наймарк, Линейные дифференциальные операторы, Наука, Физматлит, М., 1969.

[3] Н. Данфорд, Дж. Т. Шварц, Линейные операторы. Спектральная теория. Самосопряженные операторы в гилъбертовом пространстве, Мир, М., 1966.

[4] W. N. Everitt, A. Zettl, "Generalized symmetric ordinary differential expression. I: The general theory", Nieuw Arch. Wisk. (3), 27:3 (1979), 363-397.

[5] С. А. Орлов, "Об индексе дефекта линейных дифференциальных операторов", Докл. АН СССР, 92:3 (1953), 483-486.

[6] Ф. А. Неймарк, "Об индексе дефекта дифференциального оператора", УМН, 17:4 (1962), 157-163.

[7] R. B. Paris, A. D. Wood, "On the $\mathscr{L}_{2}(I)$ nature of solutions of $n$-th order symmetric differential operator and McLeod's conjecture", Proc. Roy. Soc. Edinburgh Sect. A, 90:3-4 (1981), 209-236.

[8] R. B. Paris, A. D. Wood, Asymptotics of high order differential equations, Pitman Res. Notes Math. Ser., 129, Longman, Harlow; Wiley, New York, 1986.

[9] Х. Трибель, Теория интерполяции, функциональные пространства, дифференциальные операторы, Мир, М., 1980.

[10] Ю.В. Орочко, "Индексы дефекта одночленного симметрического дифференциального оператора четного порядка, вырождающегося внутри интервала", Матем. сб., 196:5 (2005), 53-82.

[11] К.А. Мирзоев, "О теореме Орлова об индексе дефекта дифференциальных операторов”, Докл. РАН, 380:5 (2001), 591-595.

[12] Ф.Р. Гантмахер, Теория матрии, Гостехиздат, М., 1953.

[13] Э. А. Коддингтон, Н. Левинсон, Теория обыкновенных дифференииальных уравнений, ИЛ, М., 1958.

[14] Э. Л. Айнс, Обыкновенные дифференциалъные уравнения, ОНТИ, Харьков, 1939.

\section{И. Н. Долгих (I. N. Dolgikh)}

Поморский государственный университет

им. М. В. Ломоносова, г. Архангельск

E-mail: dolgih.irina@pomorsu.ru

\section{К. А. Мирзоев (К. А. Mirzoev)}

Московский государственный университет

им. М. В. Ломоносова

E-mail: akm10@yandex.ru
Поступила в редакцию 01.09.2005 